\title{
Ärztenetzwerk-Strategien für 2016? Es gibt sie wirklich!
}

\section{Daniel Maurer}

Dr. med., Präsident Ärztenetzwerk Thurgau

\author{
Der 18. Managed Care Workshop in Scuol fand vom 3. bis 5. Dezember 2015 statt.
}

Managed Care und VUCA-Welt - das war die Einleitung des Artikels zum Workshop 2014 [1]. Die VUCA-Welt (Volatil/Ungewiss/Complex/Ambivalent) hat sich im 2015 weiter akzentuiert: sozial, politisch, ökonomisch und nicht zuletzt im Gesundheitswesen.

\section{Wo steht Managed Care?}

Entwicklungen im Jahr 2015 entsprechen dem raschen Verlauf der VUCA-Welt. Positive wie negative Entwicklungen gehen Hand in Hand. Die Dachorganisation der Ärztenetzwerke medswiss.net etabliert sich immer besser und vertritt die Anliegen gesamtschweizerisch. Auch mfe (Haus- und Kinderärzte Schweiz) und SGAIM haben sich formiert und positioniert. Das gibt Rückhalt auf allen Ebenen. Die Ärztenetzwerke sind sich ihrer Verantwortung jetzt und in Zukunft bewusst. Es geht um den Erhalt der Hausarztmedizin und der integrierten Versorgung im Rahmen von Managed Care. Für diese Aufgabe werden aber klinische Patientendaten und Qualitätsbeweise benötigt. Aktuell sind Daten kaum bis gar nicht vorhanden. Ein weiteres Problem ist

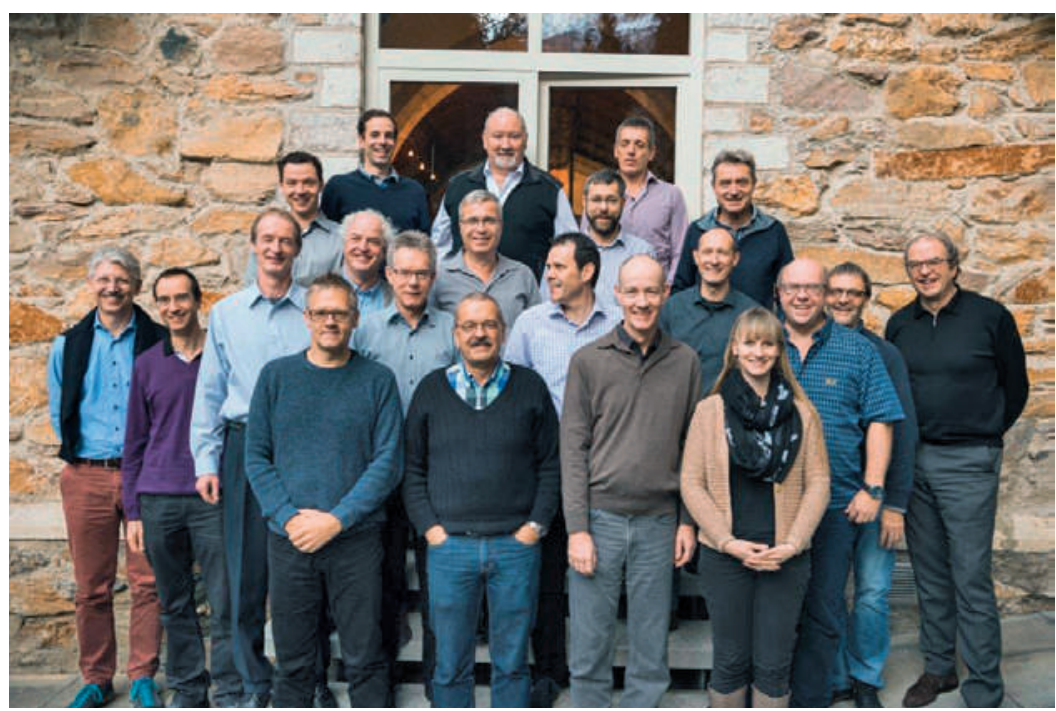

Teilnehmer des Managed Care Workshop Scuol 2015.

(Foto: Dr. Hansjörg Schlegel) der Hausarztmangel. Getroffene Massnahmen werden sich erst in Jahren auswirken. Hier können die Ärztenetzwerke Angebote machen: garantierte Grundversorgung in der Zukunft. Der Kauf einer ÄrztepraxisKette durch einen Detailhandel-Grossisten stimmt unglücklich: Monetäre Interessen oder Patientenwohl? Das Auftreten von Investoren kann nur zeigen: Der Gesundheitsmarkt ist für Investoren lukrativ. Im Jahr 2016 zeigt sich eine Renaissance von Listenmodellen: Krankenkassen sind erfindungsfreudig. Krankenkassen als Trittbrettfahrer (Leistungen beziehen ohne finanzielle Beteiligung) sind ein Ärgernis. Das neueste Produkt: Versicherungsmodell mit der Verpflichtung, als medizinische Erstanlaufstelle eine Partnerapotheke zu kontaktieren. Prämieneinsparungen bis 19\% sind möglich. Für viele Patienten und gesunde Nochnicht-Patienten ist der finanzielle Anreiz gross. Störend ist, dass bei solchen Versicherungsmodellen Erfolgszahlen zu Kostenunterschieden erst nach fünf Jahren erbracht werden müssen: genügend Zeit, erst dann die Rabatte anzupassen oder dies nicht zu tun, mit Ersatz durch wieder ein neues Produkt. Die Ärztevertretung in den politischen Gremien ist zahlenmässig knapp bis rückläufig. Ein grosses Manko! Der Patient, grösster Kostentreiber im Gesundheitswesen, will sofortig verfügbare, jederzeit abrufbare Informationen. Diese findet er im Internet. Die positiven Aspekte des Internets müssen erfasst und sinnvoll adaptiert übernommen werden. Ärztenetzwerke und Netzärzte sprechen Patienten betreffend Managed Care zu wenig an; dies sowohl für positive Aspekte als auch hinsichtlich negativer Entwicklungen.

\section{Zukunftsstrategien für Managed Care}

In Diskussionen, Workshops und Präsentationen wurden die dargestellten Themenblöcke aufgearbeitet. Wie schon früher zeigte sich hier die Diversität der Ärztenetzwerke; was im einen Netzwerk gut möglich ist, ist im anderen kaum durchsetzbar. Eine Problemlösung kann nicht für alle Netzwerke flächendeckend über- 
nommen werden. Passivität, mangelnde Flexibilität und Innovation; das könnte den Netzwerken die Substanz kosten. Ohne genügende Anzahl MC-Versicherte ist ein Netz nicht überlebensfähig. Hier zeigt sich auch, dass die alten Netzwerk-Verträge mit Capitation zunehmend sinkende Rentabilität haben. Wie müssen sich nun Ärztenetzwerk und Netzärzte verhalten? Die Netzwerkarbeit muss konsequent geleistet werden (Qualitätsmanagement, Triage der Patienten, Gate-Keeping, Kostenkontrolle). Der Mehrwert von Ärztenetzwerken muss aufgezeigt und publik gemacht werden. Der Netzwerkarzt muss seine Hausarztpatienten bewusst ansprechen (gute und schlechte Kassen, Nutzen von Managed Care). Durch optimale Vernetzung sollen Behandlungspfade vereinfacht und Kostenfolgen optimiert sowie eHealth als Hilfsmittel sinnvoll einbezogen werden. Auf nationaler Ebene muss medswiss.net mit Konsequenz überregionale Themen, Projekte und Beschlüsse durchsetzen. Managed Care soll als hochwertig und nutzvoll präsentiert werden. Dies muss mit Daten objektiv bewiesen sein. Aus Sicht von Managed Care soll auch ein Rating über die Qualität von Krankenkassen durchgeführt werden. Es soll bekannt sein, welche Kassen netzwerkkompatibel und für Managed Care der richtige Partner sind. Danach sind auch neue Ärztenetzwerk-Verträge zu entwickeln und abzuschliessen. Nicht mehr Capitation als Basis, sondern Zugang zu Grundversorgung, Qualität und optimale Behandlungspfade sollen entsprechend honoriert werden. Organisationen wie mfe und SGAIM müssen weiterhin für MC-Anliegen gewonnen werden. Mit den Ärzten in den politischen Gremien und auch im Bundesrat müssen Anliegen angebracht und positive wie negative Entwicklungen aufgezeigt werden. Jeder Hausarzt muss als Care Manager dafür kämpfen, dass die Patienten in Zukunft nicht nur aus kommerziellen Interessen betreut werden und dass die Hausarztmedizin durch die Hausärzte selbst definiert und festgelegt wird. Intellektuelle Leistungen sollen über technischen Leistungen stehen (auch bei Abgeltungen in Ärztenetzwerkverträgen). Qualität in der Hausarztmedizin und in den Netzwerken muss durch die Hausärzte und durch die Netzwerke selbst bestimmt werden.

\section{Referate zu Projekten und Analysen}

Ein Grossteil der Referate und Analysen beschäftige sich mit den direkten Partnern wie Patienten, Krankenkassen, Behörden und Politik. Qualität im Ärztenetzwerk, eHealth und Medikation waren weitere Themen. Organisationen wie medswiss.net und mfe
(Kinder- und Hausärzte Schweiz) berichteten über ihre aktuellen Analysen und Aktivitäten. Hervorzuheben war das Referat von Frau Prof. Dr. A. Belliger über das Thema "Connected Health": Sie zeigte den zunehmenden Einfluss der digitalen Veränderungen auf. Internet als Datenbank und Nachschlagwerk. GesundheitsApps zur Gesundheitsanalyse und -vorsorge. Die Bevölkerung ist online; sie will rasch und unverbindlich und zu jeder Zeit alle Informationen zu einem Thema; und dies mit maximaler Vernetzung.

\section{Fazit}

Die äusseren Umstände und Einflüsse ändern sich rascher, als dass sich ein Ärztenetzwerk genügend schnell anpassen kann. Die Netzwerke sind aktuell vor allem in der Verteidigungsposition. Sie sind zu fest systemorientiert, zu zentral organisiert, zu traditionell. Neue Ideen, neue Strategien, neue "Wege» sind gefragt. Innovation und Flexibilität und kein Warten, bis es anderswo gemacht wird. Somit steht das Handlungs-Szenario fest: Netzwerkarbeit muss konsequent geleistet werden. Neue Entwicklungen müssen rasch erfasst, ausgewertet und dementsprechend Anpassungen veranlasst werden. Daten zu Patienten, Therapie und Kosten müssen aktiv gesammelt werden. Damit werden Nutzen und Erfolg von Managed Care objektiv aufgezeigt und bewiesen. Negative Entwicklungen müssen opponiert und verhindert werden. Der wichtigste Partner von Managed Care ist der Patient (und auch der gesunde Noch-nicht-Patient). Er soll direkt angesprochen, informiert und partnerschaftlich an Bord genommen werden. Alle Entscheide und Handlungen müssen zu Gunsten der Hausarztpatienten und nicht zu deren Lasten sein. Ein Ranking betreffend Netzwerk-Kompatibilität von Krankenkassen ist zu erstellen und zu publizieren. Diese übergeordnete Aufgabe fällt medswiss.net zu. Eine Image-Kampagne zu Gunsten von Managed Care und der Ärztenetzwerke soll von medswiss.net geführt werden. Auch hier ist das Datenmaterial zur Beweisführung gegenüber Krankenkassen und politischen Gremien nötig. All dies gelingt aber nur, wenn jeder einzelne Netzwerkarzt gegenüber seinen ihm vertrauenden Patienten auch Netzwerk-Themen darstellt, diskutiert und erklärt. Der Patient soll für Managed Care sensibilisiert werden, und er soll zum Partner und Mitstreiter gemacht werden.

Der Workshop wurde moderiert von Dr. sc. nat. ETH Hansjörg Schlegel und unterstützt von der Firma Novartis Pharma Schweiz AG, vertreten durch Herrn Lorenz Borer. 